\title{
Fault Tolerant Mechanism of Bio-inspired Adaptive Routing System
}

\author{
Akiyuki Iwasaki Tadasuke Nozoe Takashi Kawauchi Masahiro Okamoto \\ Laboratory of Bioinformatics, Graduate School of Systems Life Sciences, \\ Kyushu University, \\ Fukuoka, \\ 812-8581, Japan, (Tel) +81-92-642-2899 \\ \{a-iwasaki, nozoe, kawauchi, okahon\}@brs.kyushu-u.ac.jp
}

\begin{abstract}
The routing algorithm of SPF (Shortest Path First) is widely distributed in the Internet. Since this routing algorithm is designed in order to improve throughput of each packet, it is not suitable for averaging load balance in the network. On the contrary, metabolic networks in the cell can realize load balance and achieve fault-tolerance by using enzymatic feedback mechanism. That is, a metabolic pathway in the cell is composed of a lot of enzymatic reaction steps in which biochemical reactant (substrate) is converted to the product by unique enzyme, and the product of a late step frequently acts as an inhibitor of the first committed step in this pathway (feedback control). This way, the end product of a pathway controls its own synthesis and prevents useless accumulation of intermediates and of end product.

Recently, by mimicking enzymatic feedback mechanism in the cell, we have designed a fault-tolerant adaptive routing algorithm to avoid the partial and time-variant congestions in the network. We evaluated and compared the proposed algorithm with SPF and ECMP (Equal Cost Multi-path Protocol) by using the simulation of test data. This study shows the mechanism how the proposed algorithm can remarkably improve both latency, load balance and fault tolerance.

Since there are enormous numbers of nodes in the Internet, however, it is difficult to replace all existing nodes to the proposed nodes. We shall next propose an efficient method for the allocation of adaptive nodes in random and a scale-free network composed of 100 nodes. And we describe the method of the proposed algorithm without useless. Examined the time-variant traffic at each node, and only focused on around 10\% top ranked heavy-traffic nodes, we replace such nodes to our proposed adaptive nodes. By doing this, we could design a fault-tolerant adaptive routing, which can dynamically average load-balance within the network.
\end{abstract}

\section{Keywords}

Routing algorithm, Bio-inspired system, Load balance.

Permission to make digital or hard copies of all or part of this work for personal or classroom use is granted without fee provided that copies are not made or distributed for profit or commercial advantage and that copies bear this notice and the full citation on the first page. To copy otherwise, or republish, to post on servers or to redistribute to lists, requires prior specific permission and/or a fee.

Bionetics '08, November 25-28, 2008, Hyogo, Japan

Copyright 2008 ICST 978-963-9799-35-6.

\section{INTRODUCTION}

The SPF algorithm is employed as typical routing algorithm in the Internet. However the SPF causes network congestion because all data packets are routed via the same shortest path to the destination although some redundant paths in the network exist [1]. In addition, SPF is vulnerable to network failure because it does not have the adaptability to the fluctuation in traffic. The Equal Cost Multi Path (ECMP) routing [2], [3] was proposed to achieve load distribution, which splits load equally over multiple equal cost paths. However ECMP splits load only over equal cost paths, so it is not necessarily distributing load sufficiently.

Recent studies on the Internet topology indicate that AS-level and router-level networks belong to a scale-free network with a power-law degree distribution [4]. In the past, most studies on the Internet had modeled the Internet topology either as regular network, such as a square lattice, or as completely random network, such as Waxman model [5]. However, recently, there have been many studies concerning properties of scale-free networks such as load distribution [6], routing [7], [8], path finding [9], tolerance to congestion failure [10] and optimal network structure for packet flow [11]. Thus, many researchers have focused on the relation between the Internet performance and scale-free properties. So we have to design a routing algorithm taking into account the scale-free property of the Internet.

There is a metabolic network in the cell, which is composed by biochemical reactions. In contrast to the Internet, the metabolic network achieves load balance and fault-tolerance with using feedback control mechanism such as competitive inhibition even when unexpected external perturbations are added to the network. That is, the feedback control mechanism should so-called play an important role in load balance and fault-tolerance in the network. In addition, it was reported that the metabolic network also belongs to a scale-free network [12] and has a backbone network [13]. That is, it is believed that the structure of metabolic network is similar to that of the Internet. Recently, we have focused on these properties of the control mechanism in the metabolic networks and have proposed the adaptive routing algorithm mimicking the feedback mechanism to realize load balance and fault- tolerance in the Internet [14]. First of all, in this paper, we describe the proposed adaptive routing algorithm mimicking competitive inhibition, one of the typical negative feedback mechanisms, and show the effectiveness of this algorithm for load balance and fault-tolerance thorough simulation experiments. Since there are enormous numbers of nodes in the Internet, it is difficult to replace all existing nodes to the proposed nodes. In 
this paper, we also shall propose an efficient method for the allocation of adaptive nodes in a scale-free network.

\section{COMPETITIVE INHIBITION}

A metabolic pathway is composed of a lot of enzymatic reaction steps in which biochemical reactant (substrate) is converted to the product by unique enzyme. In metabolic pathways, the product of a late step frequently acts as an inhibitor of the first committed step in this pathway (negative feedback control). This way, the end product of a pathway controls its own synthesis and prevents useless accumulation of intermediates and of end product. There are several negative feedback control mechanisms in the cell. In this study, we employ a competitive inhibition which is one of a typical negative feedback control mechanism [15].

A competitive inhibition is a form of inhibition, which prevents binding of the substrate to enzyme by binding inhibitor to enzyme. When the inhibitor $\mathrm{B}$ is assumed to control the rate velocity of substrate A with competitive inhibition, the rate velocity of A can be mathematically written as follows:

$$
\frac{d[A]}{d t}=-\frac{V[A]}{K_{m}\left(1+[B] / K_{i}\right)+[A]}
$$

where $[A]$ and $[B]$ are value of the substrate and the product, respectively. $V$ represents maximum velocity of enzyme activity, $K_{m}$ is the value of substrate giving $0.5 \mathrm{~V}, K_{i}$ is the feedback inhibition coefficient.

\section{PROPOSED ALGORITHM}

In the case of accumulation of $\mathrm{B}$, the absolute value of the term in the right-side of (1) becomes to be small. Since the B is the end product of the pathway, we can easily consider that the accumulation of B corresponds to be "congestion" of the pathway; the absolute value of the term in the right-side of (1) represents metric of "congestion” from the view point of network routing. In this study, we evaluate congestion of neighbor node $B_{j}$ of the sending node A with use of (2), which is equivalent to (1).

$$
\begin{gathered}
c_{j}=p_{j} / V_{\max } \\
p_{j}=\frac{V_{j}[a]}{K_{m}\left(1+\left[b_{j}\right] / K_{i}\right)+[a]} \\
V_{\text {max }}=\max \left(V_{j}\right)
\end{gathered}
$$

where $[a]$ and $\left[b_{j}\right]$ are the total of reserved sending packet size (byte) to the next-hop node in buffer at sending node $\mathrm{A}$ and neighbor node $B_{j}$, respectively. $V_{j}$ is the bandwidth of link between $\mathrm{A}$ and $B_{j} . K_{m}$ and $K_{i}$ are the arbitrary coefficients. The value of $c_{j}$ in (2) decreases with the increase in the value of $\left[b_{j}\right]$. Furthermore, we define the following evaluation function:

$$
f_{j}=(1-\alpha) c_{j}+\alpha\left(\frac{\text { MaxHop }-h_{j}}{\text { MaxHop }}\right)
$$

where $c_{j}$ is the metric of congestion computed from (2). The $h_{j}$ is the number of hops from node $b_{j}$ to the final destination node and it is computed from routing table at the sending node A. MaxHop is the maximum value of all $h_{j}$, and $\alpha$ is arbitrary weighting coefficient between 0 and 1 . When $\alpha=1$, the proposed algorithm corresponds to SPF. If the packet arrives at a node other than destination node of this packet, our algorithm computes the evaluated value for all neighbor nodes by using (5). Among all neighbor nodes, our algorithm determines the next node with having the maximum evaluated value of (5), and sends the packet to the sending buffer for the next node.

Each node periodically sends the control packets to the neighbors and $\left[b_{j}\right]$ in (3), total of reserved sending packet size at node $B_{j}$, can be updated. It is called ADAPTIVE routing algorithm after this.

\section{ROUTING SIMULATION}

To evaluate performance of proposed algorithm, we have performed the following soft-simulator experiment. And we adopted packet latency as an evaluation metric of the routing algorithm, which indicates the time until arriving from the source node to destination node. If the latency of the proposed algorithm is smaller than those of conventional routing algorithms, the proposed algorithm is judged more excellent than other ones.

In addition, to verify robustness to the network failure, we cause a network down, and changing latency at that time was observed.

\subsection{Simulation Condition}

Barabasi-Albert scale-free network model [16] was used for simulation. This network topology is generated by using topology generator BRITE [17]. The total node in the network is set to 100 . In addition, the numbers of links per node is set to 2 . Total links are 197.And all pairs of nodes are connected by a full duplex link of bandwidth $100[\mathrm{Mbps}]$.

When a data packet arrives at the destination node, the packet is sent to the buffer connecting to the end host. Except for destination nodes, each packet is sent to the buffer connecting the neighbor node with maximum evaluated value defined by equation (5). For simplicity, all nodes use the same set of parameters: $K_{m}=50000, K_{i}=500$ and $\alpha=0.75$.

Total number of generated data packet is 10000 in simulation. Each data packet size is 1024 [byte], which is generated at every interval time between $100[\mu \mathrm{s}]$ and $400[\mu \mathrm{s}]$. The transferred destination node for data packet is randomly selected when the packets are generated. We do not consider packet loss. Each node informs all neighbor nodes of the total of reserved sending packet size $\left(b_{j}\right)$ at the node by sending control packets at every $150[\mu \mathrm{s}]$. The control packet size is 64[byte]. To generate these control packets, we describe basic network traffic on simulation network. At the start of simulation, all nodes generate the routing table by using Dijkstra algorithm [18]. 


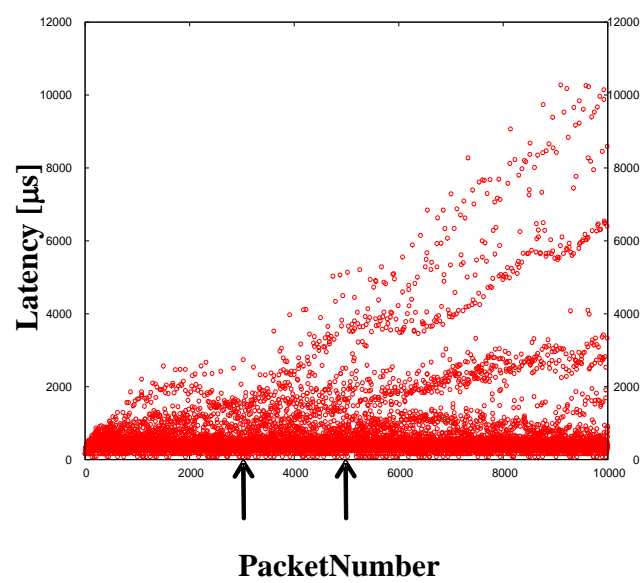

(a) SPF

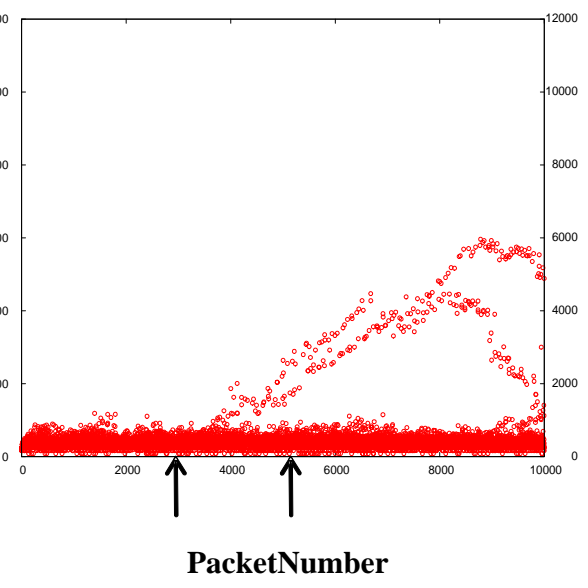

(b) ECMP

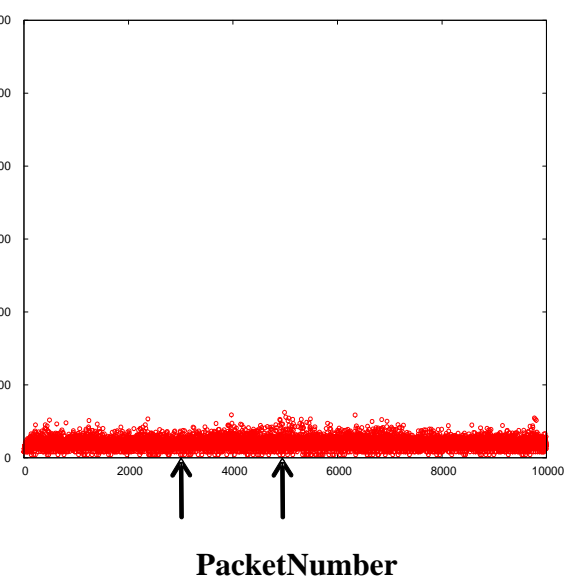

(c) ADAPTIVE

Figure.1 Latency profile in case of failure in the network.

First allow indicate the starting time of network failure and second one is the recovering time of network failure.

Table.1 Maximum and minimum, average, and standard deviation of latency shown in Figure.1

(a) SPF

\begin{tabular}{lr}
\hline Max. latency[ $\mu \mathrm{s}]$ & 10278.80 \\
Min. latency[ $\mu \mathrm{s}]$ & 81.92 \\
Ave. of latency[ $\mu \mathrm{s}]$ & 714.45 \\
Std. dev. & 1029.36 \\
\hline
\end{tabular}

(b) ECMP

\begin{tabular}{lr}
\hline Max. latency[ $\mu \mathrm{s}]$ & 5936.48 \\
Min. latency[ $\mu \mathrm{s}]$ & 81.92 \\
Ave. of latency[ $\mu \mathrm{s}]$ & 467.15 \\
Std. dev. & 564.30 \\
\hline
\end{tabular}

(c) ADAPTIVE

\begin{tabular}{lr}
\hline Max. latency[ $\mu \mathrm{s}]$ & 1243.32 \\
Min. latency[ $\mu \mathrm{s}]$ & 81.92 \\
Ave. of latency[ $\mu \mathrm{s}]$ & 394.19 \\
Std. dev. & 135.41 \\
\hline
\end{tabular}

In this study, we assumed path failures which occur for bursts of traffic such as Denial of Service (DoS) attacks. At the time of $10000[\mu \mathrm{s}]$ during simulation, bandwidths of randomly selected 10 links are narrowed down from 100 [Mbps] to 50[Mbps], and at simulation time of $20000[\mu \mathrm{s}]$, all these failures are completely recovered. Under these conditions we examined fault-tolerance ability of our ADAPTIVE routing algorithm comparing with SPF and ECMP

\subsection{Evaluation of packet latency}

Figure.1 shows the latency profile with data packet (the number of data packets is 10000) by using SPF, ECMP and the proposed algorithm (ADAPTIVE). The abscissa and the ordinate in the Figure.1 represent data PacketNumber and latency ( $\mu$ s), respectively. Table.1 summarizes the results of Figure.1; maximum latency, minimum latency, average of latency, and standard deviation in SPF, ECMP, and ADAPTIVE routing. SPF provides the increase in the latency with time because some shortest paths between end host pairs overlap other shortest paths, which leads to the congestion on the paths. As shown in Fig.1 (b), ECMP hardly provides the increase in latency with time, however, we can confirm disturbance of latency between PacketNumber $=3000$ and PacketNumber $=5000$. On the contrary, the proposed algorithm does not bring the increase in the latency. This result shows that the ADAPTIVE is able to improve latency between end hosts.

\section{EFFECT OF ADAPTIVE ROUTING TO THE NETWORK}

Figure.1 indicates the proposed algorithm can improve data packet latency. And ADAPTIVE routing has robustness or fault tolerance against the network failure. So, in this section, we shall show the mechanism how ADAPTIVE routing can remarkably improve both latency, load balance and fault tolerance.

Table.2 The value of $\alpha$ in to each group

\begin{tabular}{cccccc}
\hline Group & Ex1 & Ex1 & Ex3 & Ex4 & Ex5 \\
\hline LABEL1 & 1.0 & 1.0 & 1.0 & 1.0 & 0.75 \\
LABEL2 & 1.0 & 1.0 & 1.0 & 1.0 & 0.75 \\
LABEL3 & 1.0 & 1.0 & 1.0 & 1.0 & 0.75 \\
LABEL4 & 1.0 & 1.0 & 1.0 & 1.0 & 0.75 \\
LABEL5 & 1.0 & 1.0 & 1.0 & 1.0 & 0.75 \\
LABEL6 & 1.0 & 1.0 & 1.0 & 1.0 & 0.75 \\
LABEL7 & 1.0 & 1.0 & 1.0 & 1.0 & 0.75 \\
LABEL8 & 1.0 & 1.0 & 1.0 & 0.75 & 0.75 \\
LABEL9 & 1.0 & 1.0 & 0.75 & 0.75 & 0.75 \\
LABEL10 & 1.0 & 0.75 & 0.75 & 0.75 & 0.75 \\
\hline
\end{tabular}




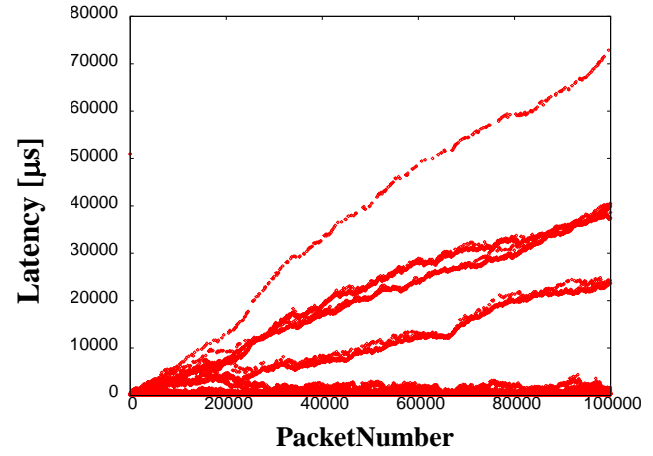

(a)SPF

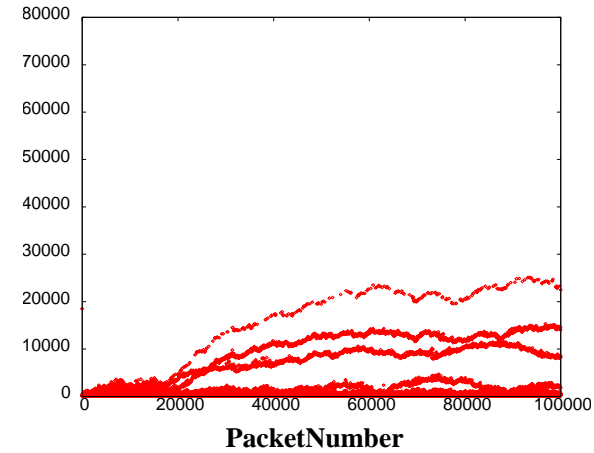

(b) $10 \%$ ADAPTIVE

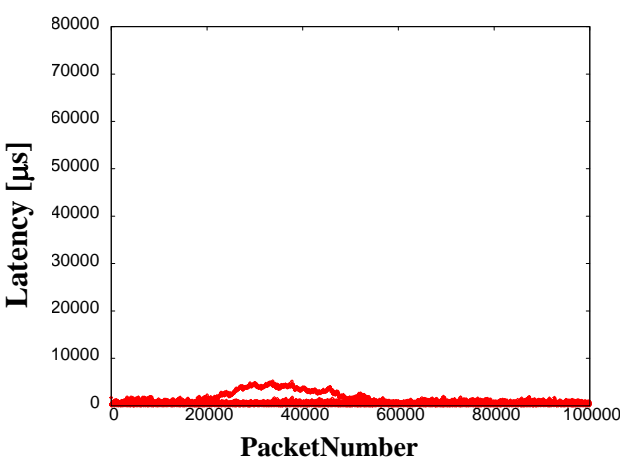

(c) $20 \%$ ADAPTIVE

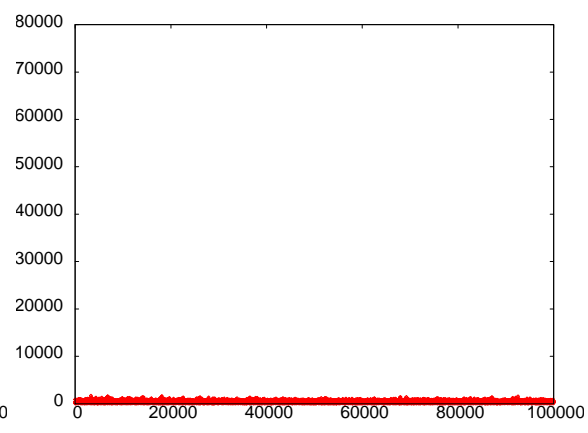

PacketNumbe

(d) $30 \%$ ADAPTIVE

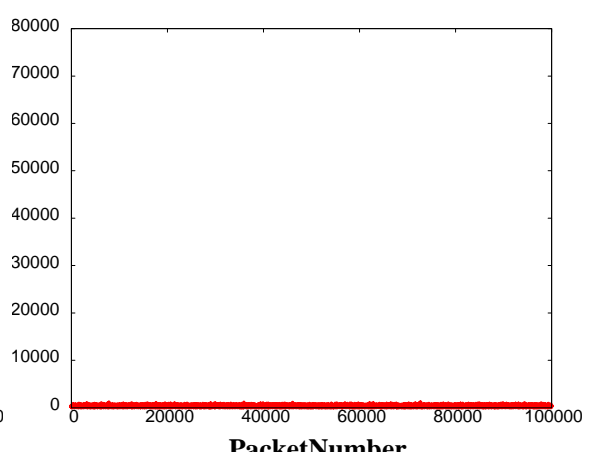

(e) All ADAPTIVE

Figure.2 Latency profile of data packet

Table.3 Maximum and minimum, average, and standard deviation of latency shown in Figure.2

\begin{tabular}{|c|c|c|c|c|c|}
\hline & SPF & 10\%ADAPTIVE & 20\%ADAPTIVE & 30\%ADAPTIVE & All ADAPTIVE \\
\hline Max. latency[ $\mu s]$ & 72871.30 & 25128.60 & 5178.72 & 1718.16 & 1253.68 \\
\hline Min. latency[ $\mu \mathrm{s}]$ & 81.92 & 81.92 & 81.92 & 81.92 & 81.92 \\
\hline Std. dev. & 6642.82 & 2447.79 & 357.25 & 143.13 & 118.44 \\
\hline
\end{tabular}

\subsection{Simulation Condition}

Simulator was performed with using the scale-free network which was same as subsection 4.2; network is composed of 100 nodes and the total number of links is 197. Total number of generated data packets is 100000 in simulation. To observe the behavior of the data packets, we divided these packets into ten groups according to the value of $\alpha$ in (5) (LABEL1 to LABEL10) The values of arbitrary coefficient $K m$ and $K_{i}$ are set to 50000 and 500 , respectively.

ADAPTIVE routing algorithm operates by the evaluation function in (5). The right side of evaluation function (5) has two terms; congestion index and distance index, respectively. And the weight of those two terms indices are controlled by the value of $\alpha$ (weight coefficient). The operation of ADAPTIVE routing is decided depending on the value of $\alpha$. In order to observe the network with ADAPTIVE routing, we set different values of $\alpha$ and classified them into ten groups (LABEL1 to 10). Table.2 shows the value of $\alpha$ in each group.
The group is divided by the equal number of packets. Each group has 10000 packets.

When the value of $\alpha$ is 1 , ADAPTIVE routing corresponds to SPF routing. It is shown that each experiment (Ex1 to Ex5) in Table. 2 is the following conditions.

(Ex1) All packets are controlled by SPF routing.

(Ex2) $10 \%$ of total number of the data packet is controlled by ADAPTIVE routing. And the others packet are controlled by SPF routing. This operation is called 10\%ADAPTIVE.

(Ex3) 20\%ADAPTIVE

(Ex4) 30\%ADAPTIVE

(Ex5) All packets are controlled by ADAPTIVE routing. This operation is called All ADAPTIVE.

Figure. 2 shows latency profile of data packets in Ex1 to Ex5. The abscissa and the ordinate represent packet number and packet latency, respectively. Table.3 summarizes the result of Figure.2; maximum, minimum, average, and standard deviation of packet latency. 


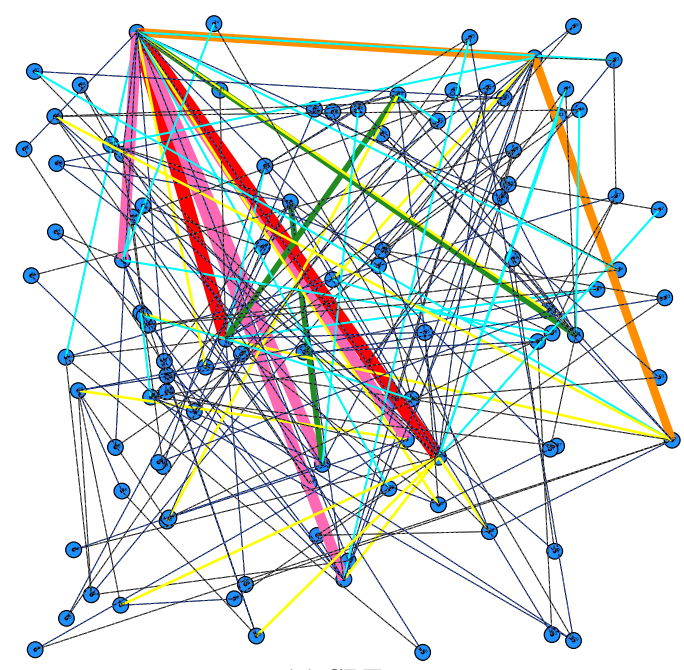

(a) SPF

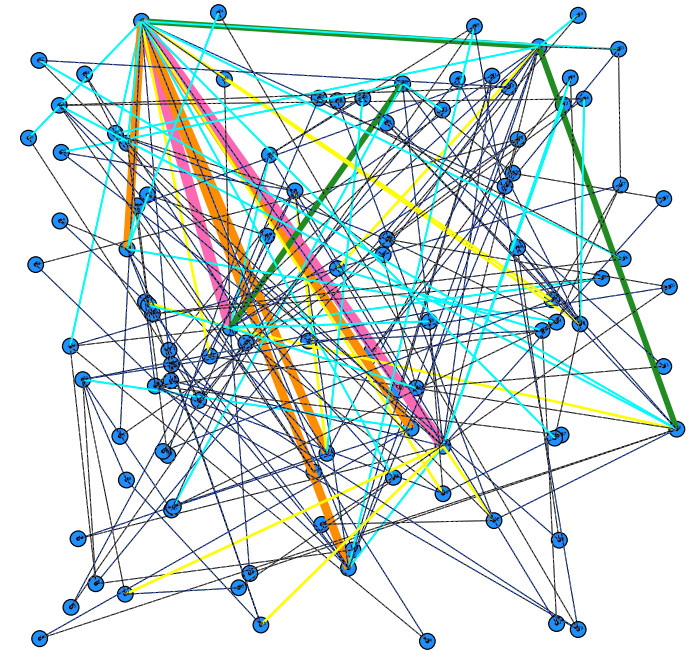

(c) 20\%ADAPTIVE

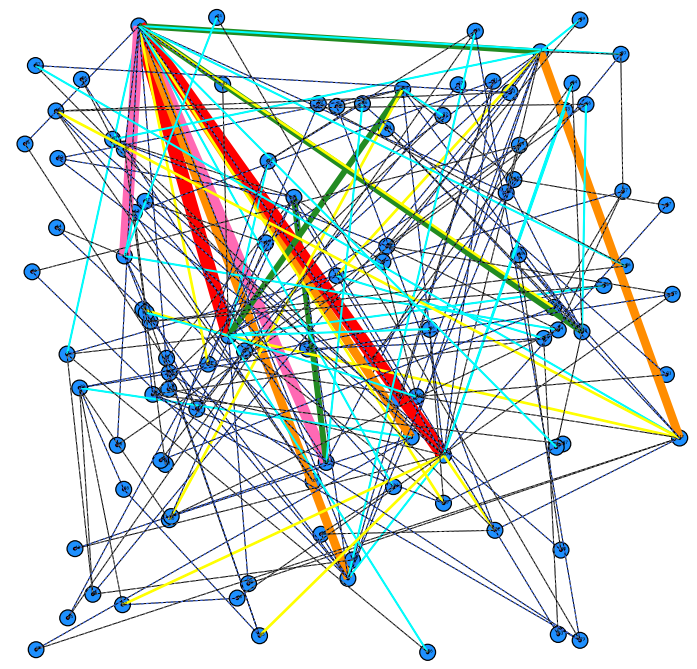

(b) 10\%ADAPTIVE

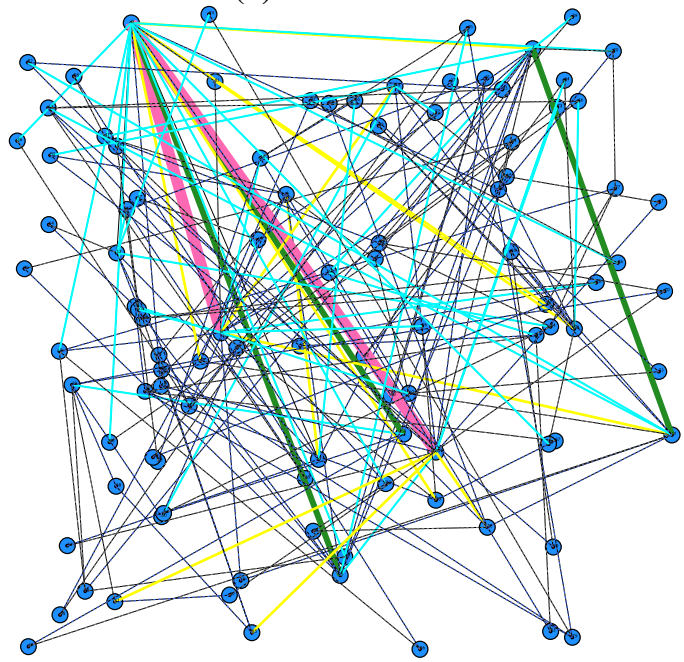

(d) $30 \%$ ADAPTIVE

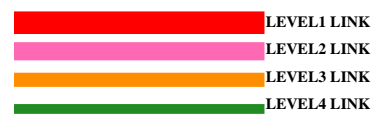

Figure.3 The number of packet passing through the link (Packet though rate) in the network

Network link level set according to Packet through rate: [LEVEL1 LINK]: Packet through rate $\geqq 3500[t i m e s]$, [ LEVEL2 LINK]: $3000[$ times] $\leqq$ Packet through rate $\leqq 3500$ [times], [LEVEL3 LINK]: 2500[times] Packet through rate 33000 [times], [LEVEL4 LINK]: 2000[times] Packet through rate $\leqq 2500[$ times]

\subsection{Evaluation of packet latency}

According to the latency profile of SPF routing algorithm (Figure.2 (a)), latency continues to grow at the latter half of packet number. On the contrary to this, in the ADAPTIVE routing (Figure.2 (e)), a large latency was not observed, which shows that ADAPTIVE routing has load balance function.

In the method of 10\%ADAPTIVE, it is shown the latency of packet improves a little (Figure.2 (b)), however, at 20\%ADAPTIVE, remarkable improvement is shown at packet latency (Figure.2 (c)).
And the result of 30\% ADAPTIVE routing (Figure.2 (d)) shows almost same as result of usual ADAPTIVE routing algorithm (Figure.2 (e)).

These result shows that the latency of the entire network can be decreased only by small number of data packets controlled by ADAPTIVE routing algorithm.

Here, we examined the mechanism how the load of the network was decreased only by small amount of packet controlled by ADAPTIVE algorithm. Figure.3 depicts the rate of packet passing through the link of network. The larger the rate of packets passing through the link is, the link between the nodes is depicted with the bolder line. 
Figure.3 (a) indicates the situation of the network with the SPF routing. SPF algorithm sets to static route to data packet. And many packets passed through the same route. As a result, many over-loaded links were generated in the network. In Figure.3 (a), there can be seen two most over-loaded links (boldest lines) between the upper left node and the lower right one.

Figure.3 (b) represents the congestion behavior with $10 \%$ ADAPTIVE routing. This routing method improves the network congestion compared with SPF, however, drastic improvement was not observed because the links of "LEVEL1 LINK (boldest lines)" remain in the network.

Figure3 (c) shows the case with 20\%ADAPTIVE routing. As shown in the figure, the link of "LEVEL 1 LINK" did not exist in the network. As a result, the bottleneck of network was removed, which load to great improvement of latency of packets.

Moreover, at the 30\%ADAPTIVE routing, the links in which the packets are forwarded with high frequency are almost disappeared. Then, the latency of packet is similar to All ADAPTIVE routing (Figure.2 (e)).

From these results, we can conclude that the increase in packet latency causes to the congestions of specific route and these congestions will be removed by introducing only small amount of ADAPTIVE packets controlled by ADAPTIVE routing algorithm; in this study, 20\%ADAPTIVE is enough.

\section{NODE CHOICE OF ADAPTIVE ROUTING}

Since it is difficult to incorporate the proposed algorithm into all nodes in a network, we have investigated on the effective selection and allocation of ADAPTIVE nodes in the network. In addition, we represented it is need not to be provided ADAPTIVE function for all packets in section 5. Nozoe et al. [19] proposed the allocation method considering with degree of a node in the network; the ADAPTIVE algorithm was adopted at the nodes with the higher node-degree.

According to them, by replacing around 20\% of top-ranked nodes with the higher degree of links to the ADAPTIVE, the network can reveal the equivalent fault- tolerant ability to that of all-ADAPTIVE-network. In order to decrease the number of the ADAPTIVE nodes as much as possible, in this study, we shall propose the alternative allocation method for selecting the ADAPTIVE nodes based time-variant traffic of packets passing through the node. Under the simulation condition shown in Figure. 1 , there are three kinds of traffic conditions such as before network failure $(0[\mu \mathrm{s}]<$ time $<10000[\mu \mathrm{s}])$, during network failure $(10000[\mu \mathrm{s}]<$ time $<20000[\mu \mathrm{s}])$ and after network failure $(20000[\mu \mathrm{s}]<$ time). We have investigated the traffic of packets passing through the node during three time periods, sequentially.

Figure. 4 shows a frequency distribution profile before, during and after network failure, respectively. The ordinate indicates the frequency (number of nodes) and the abscissa is the class of the number of packets passing through the nodes. Only focused on the nodes with having the number of packets passing through the nodes more than 300 for before and after network failure and 200 for during network failure, the ADAPTIVE algorithm was employed to these nodes. The number of the ADAPTIVE nodes is 5, 12, and 10 before, during and after network failure, respectively.
According to the frequency distribution, we select an ADAPTVE node. Selected nodes have usually higher degree (more than 17), however some node links of which is 5 was also selected [20].

Figure.5 and Table. 4 show the packet latency profile and summarized result in the case of the selected ADAPTIVE nodes network, respectively. Compared Table.4 with Table.1, the selected ADAPTIVE nodes network has higher fault-tolerant ability than that of ECMP (Table.1 (b)), and has almost equivalent ability to that of all ADAPTIVE nodes network (Table.1 (c)).

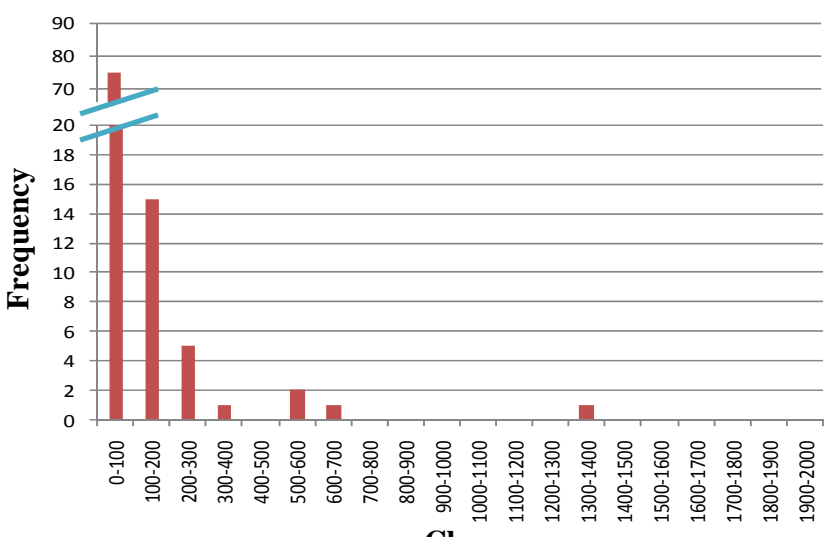

(a) Before network failure

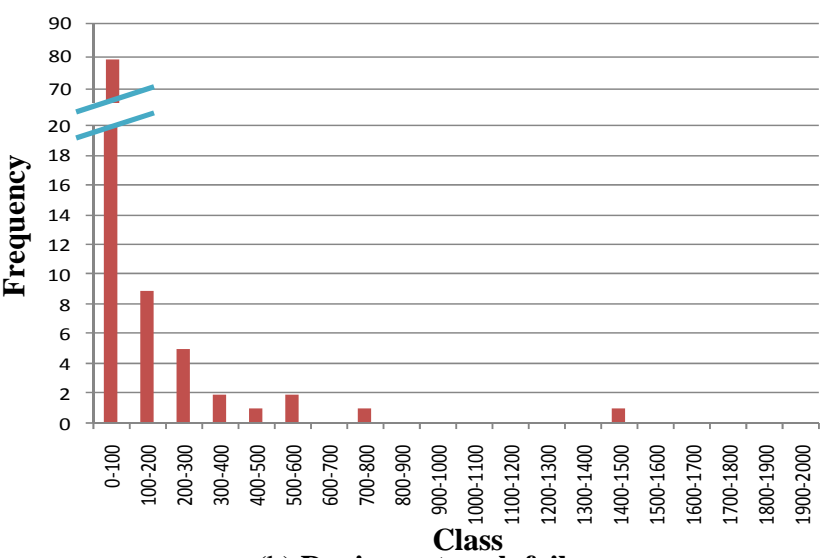

(b) During network failure

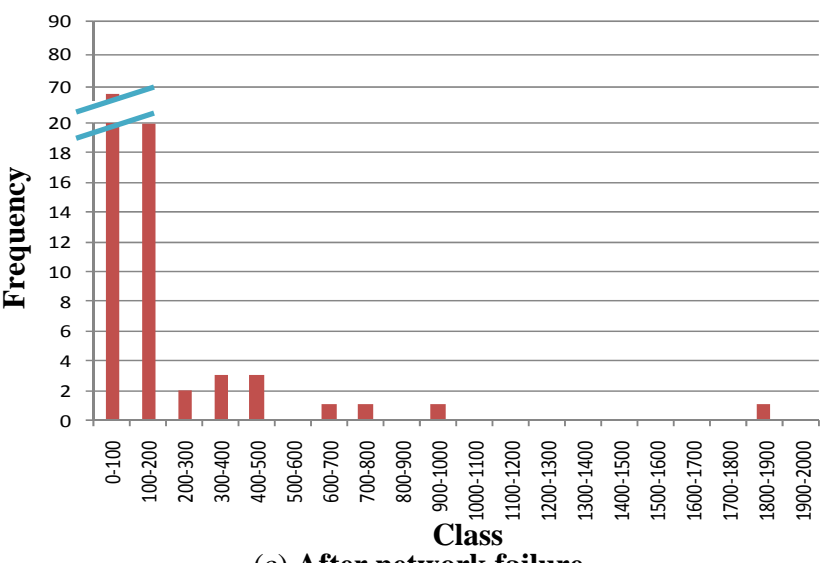

(c) After network failure

Figure.4 Frequency distribution (number of nodes) with the class of the number of packets passing through the node 
Next fixed the selected ADAPTIVE nodes, we examined the fault-tolerant ability with changing the traffic condition; change the destination node of packets. Figure. 6 and Table.5 show the latency profiles in the cases of ECMP, ALL ADAPTIVE and selected ADAPTIVE, respectively. As shown in these results, in spite of different traffic condition, the selected ADAPTIVE nodes network shows the higher fault-tolerant ability, which means network topology is predominant factor to determine the traffic condition and the selective ADAPTIVE nodes network which was nominated in a certain traffic condition also shows the higher fault-tolerant ability in other case of traffic conditions.

According to Nozoe et al. [19], the percentage of selected ADAPTIVE nodes was 20 in the same network. From the results in this study, we can reduce the selected ADAPTIVE nodes around $10 \%$ with keeping the higher fault-tolerant ability. Thus we can conclude that the allocation method for selecting the ADAPTIVE nodes based on the investigation of time-variant traffic of packets passing through the node is more effective than the allocation method considering with degree of a node in the network.

\section{DUSCUSSION}

We proposed the ADAPTIVE routing algorithm that was mimicked enzymatic negative feedback control mechanism in metabolic networks. And we evaluated the performance of the proposed rouging algorithm with SPF and ECMP routing algorithm. The simulation results with ADAPTIVE routing algorithm showed remarkable improvement of packet latency compared with the conventional routing algorithms (SPF, ECMP).

Next we proposed the efficient method for allocating and selecting the ADAPTIVE nodes in the network based on the frequency profiles where we examines the number of packets passing through the node under a certain traffic condition. By using this method, we could reduce the number of ADAPTIVE nodes up to around $10 \%$.

Since the traffic condition (congestion) changes with time, it is ideal that the ADAPTIVE nodes can be selected automatically according to the current traffic condition. Also in order to apply this method to large scale network, multi-layer ADAPTIVE network (overlay ADAPTIVE network) with having the small and indispensable number of ADAPTIVE nodes has to be designed.

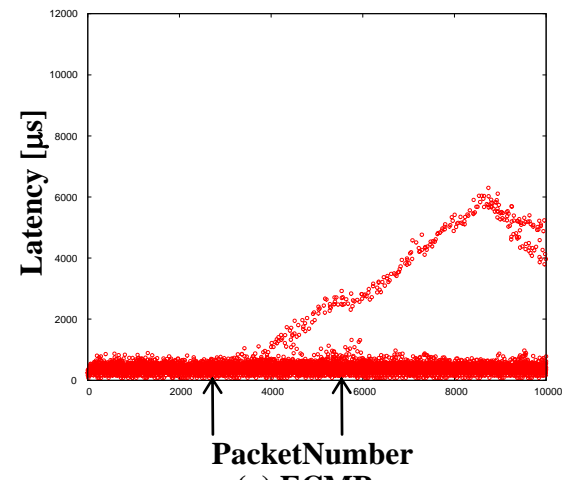

(a) ECMP

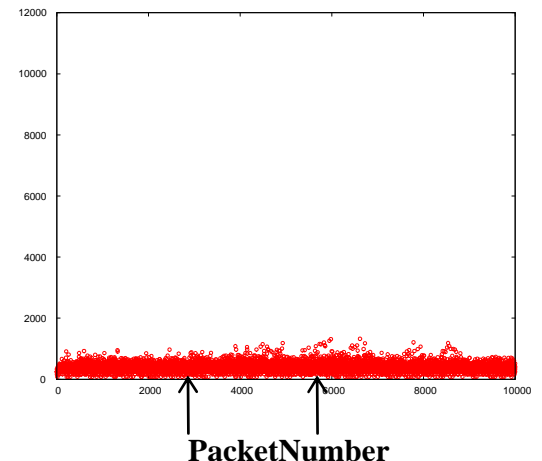

(b) All ADAPTIVE

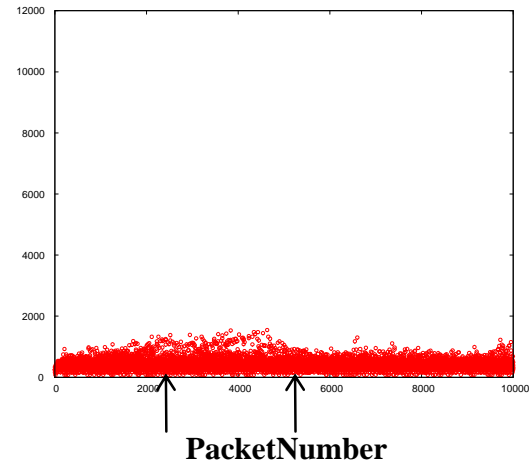

(c) Selected ADAPTIVE

Figure.6 Latency profile in case of failure in the network.

First allow indicate the starting time of network failure and second one is the recovering time of network failure. 
Table.5 Maximum and minimum, average, and standard deviation of latency shown in Figure.6

(a) ECMP

\begin{tabular}{|c|c|}
\hline Max. latency $[\mu \mathrm{s}]$ & 6298.48 \\
\hline Min. latency[ $\mu \mathrm{s}]$ & 81.92 \\
\hline Ave. of latency[ $\mu \mathrm{s}]$ & 472.96 \\
\hline Std. dev. & 626.59 \\
\hline
\end{tabular}

(b) All ADAPTIVE

\begin{tabular}{|c|c|}
\hline Max. latency[ $\mu$ s] & 1326.56 \\
\hline Min. latency[ $\mu \mathrm{s}]$ & 81.92 \\
\hline Ave. of latency[ $\mu \mathrm{s}]$ & 391.35 \\
\hline Std. dev. & 135.51 \\
\hline
\end{tabular}

(c) Selected ADAPTIVE

\begin{tabular}{lr}
\hline Max. latency[ $\mu \mathrm{s}]$ & 1544.24 \\
Min. latency[ $\mu \mathrm{s}]$ & 81.92 \\
Ave. of latency[ $\mu \mathrm{s}]$ & 420.56 \\
Std. dev. & 174.16 \\
\hline
\end{tabular}

according to the number of packet and congestion situation in the network; it is possible to produce the best situation of communication network.

Resent research says the topological structure of Internet is different from scale-free and random network [21]. So we need to simulation at another network which indicates topological structure of real network (i.e. data from CAIDA [22]). And check proposed routing algorithm can improve packet latency at that condition.

\section{ACKNOWLEDGMENTS}

This work was partially supported by the Grants-in-Aid for Scientific Research (B) (No.19300020 (MO)) from Ministry of Education, Culture, Sports, Science and Technology in Japan.

\section{REFERENCES}

[1] R. Teixeira, K. Marzullo, S. Savage and G. M. Voelker, "In Search of Path Diversity in ISP Networks," in Proc.ACM SIGCOMM Internet Measurement Conference, 2003, pp. 313-318.

[2] D. Awduche, A. Chiu, A. Elwalid, I. Widjaja, and X. Xiao, "Overview and Principles of Internet Traffic Engineering," RFC3272, 2002.

[3] T.-W. Chim, K.-L. Yeung and K.-S. Lui, “Traffic distribution over equal-cost-multi-paths," Computer Networks, vol. 49, pp. 465-475, 2005.

[4] M. Faloutsos, P. Faloutsos and C. Faloutsos, "On PowerLaw Relationships of the Internet Topology,” in Proc. ACM SIGCOMM, 1999, pp. 251-262.

[5] B. M. Waxman, "Routing of multipoint connections with graphs,” IEEE. Journal on Selected Areas in Communications, vol. 6, no. 9, pp. 1617-1622, 1988.

[6] K.-I. Goh, B. Kahng, and D. Kim, "Universal Behavior of Load Distribution in Scale-Free Networks," Physical Review Letters, vol. 87, 278701, 2001.

[7] C.-Y. Yin, B.-H Wang, W.-X Wang, T. Zhou and H.-J. Yang, "Efficient routing on scale-free networks based on local information,” Physics Letters A, vol. 351, pp. 220-224, 2006.

[8] G. Yan, T. Zhou, B. Hu, Z.-Q. Fu and B.-H Wang, "Efficient routing on complex networks,” Physical Review E, vol. 73, 046108, 2006.

[9] B.-J. Kim, C.-N. Yoon, S.-K Han and H. Jeong, "Path finding strategies in scale-free networks," Physical Review E, vol. 65, 027103, 2002.
[10] Y. Moreno, R. Paster-Satorras, A. Vazqes and A. Vespignani, "Critical load and congestion instabilities in scale-free networks," Europhysics Letters, vol. 62, no. 2, pp. 292-298, 2003.

[11] J. Ohkubo and T. Hosiguch, "Scale-free property of optimal network for packet flow by a packet routing control," Physica A, vol. 353, pp. 649-660, 2005.

[12] H. Jeong, B. Tombor, R. Albert, Z. N. Oltvai and A.-L. Barabasi, "The large-scale organization of metabolic networks,” Nature, vol.407, pp.641-654, 2000.

[13] E. Almaas, B. Kovacs, T. Vicsek, Z. N. Oltvai,A.-L. Barabasi, "Global organization of metabolic fluxes in the bacterium Escherichia coli,” Nature, vol. 427, pp. 839-843, 2004.

[14] T.Kawauchi, T. Nozoe, and M. Okamoto, ”Biologically Inspired Adaptive Routing by Mimicking Enzymic Feedback Control Mechanism in the Cell,” Lecture Notes in Computer Science, vol. 3853, pp. 371-378 2006.

[15] M.Okamoto, M.Takada, Y.Aso, K. Hayashi, " Steady-state approximation of enzyme activation and inhibition", Biotechnol. and Bioengineer., vol.25, pp1453-1463, 1983.

[16] A.-L. Barabasi and R. Albert, "Emergence of Scaling in Random Networks,” Science, vol. 286, pp.509-512, 1999.

[17] A. Medina, A. Lakhina, I. Matta, and J. Byers, "BRITE: An Approach to Universal Topology Generation,” in Proc. the International Workshop on Modeling, Analysis and Simulation of Computer and Telecommunication System, 2001.

[18] Dikjstra,E.W.: A note on two problem in connection with graphs, Numerische Mathematik, 1, pp269-271(1959)

[19] T.Nozoe, T. Kawauchi, and M.Okamoto, “A Bio-inspired Adaptive Routing based on Enzymatic Feedback Control Mechanism in Metabolic Networks” IMECS 2007

[20] Akiyuki Iwasaki, Tadasuke Nozoe, Takeshi Kawauchi, Masahiro Okamoto: "Design of Bio-inspired Fault-tolerant Adaptive Routing Based on Enzymatic Feedback Control in the Cell: Towards Averaging Load Balance in the Network" Frontiers in the Convergence of Bioscience and Information Technologies: FBIT, pp854-860(2007)

[21] Rami Cohen, Danny Raz, "The Internet Dark Matter - on the Missing links in the AS Connectivity Map", Proceedings- IEEE INFOCOM, article no 4146887, 2006

[22] Cooperative Association for Internet Data Analysis (CAIDA), http://www.caida.org. 